\title{
Value of Early Post-Operative Growth Hormone Testing in Predicting Long-Term Remission and Residual Disease after Transsphenoidal Surgery for Acromegaly
}

\author{
Yi Yuen Wang ${ }^{a, b}$ Mueez Waqar ${ }^{a, c}$ Ahmed Abou-Zeid ${ }^{a, d}$ Tara Kearney $^{\mathrm{e}}$ \\ Carmela Caputo $^{f}$ Julian Davis ${ }^{g}$ Peter Trainer $^{\text {h }}$ Claire Higham $^{\text {h }}$

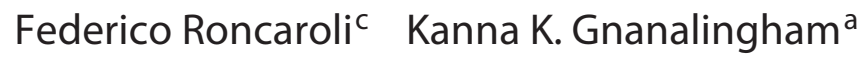

\begin{abstract}
aDepartments of Neurosurgery, Greater Manchester Neurosciences Centre, Salford Royal Foundation Trust (SRFT), Manchester Academic Health Sciences Centre, Manchester, UK; ${ }^{b}$ Department of Neurosurgery, The University of Melbourne, St Vincent's Hospital, Fitzroy, VIC, Australia; 'Division of Neuroscience and Experimental Psychology, School of Biology, Faculty of Biology, Medicine and Health, Geoffrey Jefferson Brain Research Centre, University of Manchester, Manchester, UK; ${ }^{d}$ Department of Neurosurgery, Ain Shams University, Cairo, Egypt; ${ }^{\text {eDepartment }}$ of Endocrinology, Greater Manchester Neurosciences Centre, Salford Royal Foundation Trust (SRFT), Manchester Academic Health Sciences Centre, Manchester, UK; ' Department of Endocrinology, The University of Melbourne, St Vincent's Hospital, Fitzroy, VIC, Australia; ' Department of Endocrinology, Manchester University NHS Foundation Trust, and Faculty of Biology, Medicine \& Health, Manchester Academic Health Sciences Centre, University of

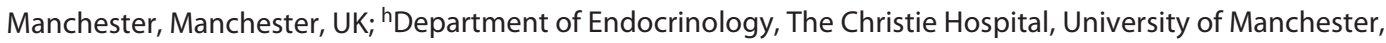
Manchester Academic Health Science Centre, Manchester, UK
\end{abstract}

\section{Keywords}

Acromegaly · Growth hormone - Oral glucose tolerance test · Insulin-like growth factor-1 · Remission · Early tests

\begin{abstract}
Introduction: Surgical remission for acromegaly is dependent on a number of factors including tumour size, invasiveness, and surgical expertise. We studied the value of early post-operative growth hormone $(\mathrm{GH})$ level as a predictor of outcome and to guide early surgical re-exploration for residual disease in patients with acromegaly. Methods: Patients with acromegaly undergoing first-time endoscopic transsphenoidal surgery between 2005 and 2015, in 2 regional neurosurgical centres, were studied. Insulin-like growth factor-1 (IGF-1), basal GH (i.e., sample before oral glucose), and $\mathrm{GH}$ nadir on oral glucose tolerance test (OGTT)
\end{abstract}

karger@karger.com www.karger.com/nen

Karger $\frac{1}{\%}$
C 2021 The Author(s).

Published by S. Karger AG, Basel

This is an Open Access article licensed under the Creative Commons Attribution-NonCommercial-4.0 International License (CC BY-NC) (http://www.karger.com/Services/OpenAccessLicense), applicable to the online version of the article only. Usage and distribution for commercial purposes requires written permission. were tested at various time points, including $2-5$ days postoperatively. Definition of disease remission was according to the 2010 consensus statement (i.e., $\mathrm{GH}$ nadir $<0.4 \mu \mathrm{g} / \mathrm{L}$ during an OGTT and normalized population-matched IGF-1). Forward stepwise logistic regression was used to determine factors associated with remission. Results: We investigated 81 consecutive patients with acromegaly, 67 (83\%) of which had macroadenomas and 22 (27\%) were noted to be invasive at surgery. Mean follow-up was $44 \pm 25$ months. Overall, surgical remission was achieved in $55(68 \%)$ patients at final follow-up. On univariate analysis, the remission rates at the end of the study period for patients with early post-operative GH nadir on OGTT of $<0.4(N=43)$, between 0.4 and 1 $(N=28)$, and $>1 \mu \mathrm{g} / \mathrm{L}(N=8)$ were 88,54 , and $20 \%$, respectively. Similar results were seen with basal $\mathrm{GH}$ on early postoperative OGTT. On multivariate regression analysis, pre-operative IGF-1 (odds ratio of 13.1) and early post-operative 
basal GH (odds ratio of 5.0) and GH nadir on OGTT (odds ratio of 6.8) were significant predictors of residual disease. Based on a raised early $\mathrm{GH}$ nadir and post-operative MR findings, 10 patients underwent early surgical re-exploration. There was reduction in post-operative $\mathrm{GH}$ levels in 9 cases, of which 5 (50\%) achieved long-term remission. There was an increased risk of new pituitary hormone deficiencies in patients having surgical re-exploration compared to those having a single operation (60 vs. 14\%). Conclusions: An early post-operative basal GH and GH nadir on OGTT are reliable predictors of long-term disease remission. It can be used to guide patients for early surgical re-exploration for residual disease, although there is increased risk of hypopituitarism.

(c) 2021 The Author(s).

Published by S. Karger AG, Basel

\section{Introduction}

Transsphenoidal surgery is the first-line treatment option for patients with a growth hormone (GH)-secreting adenoma. Surgical remission rates can vary between 20 and $85 \%$, depending on a number factors including tumour size, invasiveness, pre-operative GH levels, and also the criteria applied to define disease remission $[1,2]$. Surgical expertise is also an important determinant of outcome in acromegaly surgery. Remission rates improve when surgery is performed by dedicated pituitary surgeons who undertake large volume pituitary surgery [3-5].

The definition of post-operative remission of acromegaly has evolved over time, in part due to the development of more sensitive GH assays [1,2]. Following the publication of the consensus statement in 2010, biochemical remission is now defined as a GH nadir on an oral glucose tolerance test (OGTT) of $<0.4 \mu \mathrm{g} / \mathrm{L}$ and an insulin-like growth factor-1 (IGF-I) within the age- and gender-normalized reference range [2].

In the immediate post-operative period, a robust early test of disease remission is of use as, in combination with MR-imaging, it can identify patients who might benefit from early re-operation for residual tumour [5]. Early surgical re-exploration before development of post-operative reparative/scar tissue is technically less challenging. Recent studies have reported on the predictive value of an early post-operative GH nadir on OGTT on final endocrine outcome, though the exact time point that is regarded as early varies between studies [6-9]. Moreover, the reliability and timing of such early tests of the GH axis remains unclear. Early post-operative IGF-I levels on the contrary are much less useful, taking around 12 weeks to settle to a stable level [10].
In our centres, the protocol from 2005 has been to perform post-operative assessments of the $\mathrm{GH}$ axis including GH nadir on OGTT and IGF-1 levels in all acromegaly patients undergoing surgery. These assays are performed on post-operative days $2-5$ and then serially during follow-up. This practice has allowed early intervention and identification of patients with potential residual tumour. We report our experience with early $\mathrm{GH}$ axis testing as a predictive factor for long-term remission and in identifying those patients with residual tumour following endoscopic transsphenoidal resection of GH-secreting pituitary adenomas.

\section{Methods}

Institutional Review Board approval was obtained at each study centre. Consecutive patients undergoing first time endoscopic transsphenoidal surgery for a $\mathrm{GH}$-secreting adenoma between 2005 and 2015 at 2 pituitary centres in Manchester, UK and Melbourne, Australia were identified and included in this study. The demographics, clinical details, pre- and post-operative biochemical assessments, as obtained from a prospectively collated pituitary surgery database, were analysed. Preliminary results from this study have been published previously [5].

\section{GH and IGF-1 Assays}

For the OGTT, $75 \mathrm{mg}$ of glucose was administered orally following an overnight fast with concomitant glucose and GH measurements performed on plasma specimens collected at $0,30,60$, 90 , and 120 min after ingestion of the glucose load. Basal GH (i.e., sample before oral glucose) and the GH nadir during the OGTT were recorded and used in the subsequent analysis.

$\mathrm{GH}$ at both institutions was assayed by chemiluminescent immunoassay with the Siemens Immulite $2000 \mathrm{GH}$ Assay (lower detection threshold, $0.05 \mu \mathrm{g} / \mathrm{L}$; analytical sensitivity, $0.01 \mu \mathrm{g} / \mathrm{L}$ ) with standardization using the current World Health Organization (WHO) international standard (WHO IS 98/574) expressed in mass units $(\mu \mathrm{g} / \mathrm{L})[2,11]$. Assays were standardized to multiple isoforms of $\mathrm{GH}$, the majority being the $22-\mathrm{kDa}$ isoform. $\mathrm{GH}$ units were expressed in international units (mU/L) prior to June 2008, and in mass units $(\mu \mathrm{g} / \mathrm{L})$ after this time. In this study, all GH measurements are expressed in $\mu \mathrm{g} / \mathrm{L}$ with a conversion factor of $3: 1$, when results were obtained prior to 2008 .

For plasma IGF-1 concentrations, the results are expressed as a percentage of the upper limit of the normal (ULN) age- and sex-adjusted range, drawn from a statistically valid control population. In both centres, the plasma IGF-1 levels were initially assessed by chemiluminescent immunoassay, Immulite 2000 IGF-1 (Siemens Ltd.), with standardization using the WHO first IRR 87/518 and expressed in the international standard units of $\mathrm{nmol} / \mathrm{L}$. From around $2012 / 2013$, the IGF-1 samples were assessed using the chemiluminescent immunometric assay, IDS iSYS IGF-1 assay, calibrated against the new standard WHO International Standard 02/254 (Immunodiagnostic Systems Limited) and expressed in $\mathrm{ng} / \mathrm{mL}$.

All GH and IGF-1 values recorded in this study are values taken before the commencement of any medical therapy for patients 
with acromegaly. In patients on pre-operative medical therapy (notably somatostatin analogues [SSAs]), at least a 3-month drug washout period was allowed before surgery to enable meaningful interpretation of GH levels post-operatively. The diagnosis of acromegaly was confirmed biochemically by the elevation of plasma IGF-1 in conjunction with the inability to suppress serum GH during an OGTT.

\section{Radiological Assessment}

All patients underwent a dedicated MR scan of the sellar region using our institutions' pituitary protocol. Scans were performed on the Siemens 1.5 Tesla machine, acquiring sagittal (T1-weighted) and coronal (T1- and T2-weighted) 2-mm slices. Gadolinium contrast was not routinely given unless dynamic scans were performed looking for microadenomas that were not clearly visible on the standard sequences. Post-operative imaging included contrast enhancement.

Each tumour was stratified by size into micro $(<10 \mathrm{~mm})$, macro (10-25 mm), and large ( $>25 \mathrm{~mm}$ ) adenoma on maximal vertical and transverse dimensions. The degree of invasion was assessed using both the Hardy [12] and the Knosp [13, 14] grading systems. Hardy grades 3-4 and D-E and Knosp grades 3-4 were considered to be invasive adenomas.

\section{Transsphenoidal Surgery}

All transsphenoidal surgeries were performed using the endoscope by a single surgeon at each centre as previously described [15]. Surgical data were detailed in electronic operative reports, including relevant information on the surgeon's perception of tumour invasion (i.e., into cavernous sinus and/or bony clivus), degree of tumour resection, and relevant post-operative complications.

The surgical aim was a "safe" gross total resection of all tumours, except in cases where there was radiological evidence of circumferential tumour invasion around the cavernous carotid (e.g., Knosp grade 3 or 4 ). The latter patients underwent a planned surgical debulking.

\section{Post-Operative Endocrine Tests}

Early post-operative assessment of the GH axis was performed in all patients with an OGTT and IGF-1 between post-operative days 2 and 5. Based on the early post-operative OGTT results, disease remission was also categorized into the following subgroups: likely remission (i.e., early $\mathrm{GH}$ nadir $<0.4 \mu \mathrm{g} / \mathrm{L}$ ), borderline remission (i.e., early $\mathrm{GH}$ nadir $0.4-1 \mu \mathrm{g} / \mathrm{L}$ ), and unlikely remission (i.e., early GH nadir $>1.0 \mu \mathrm{g} / \mathrm{L})$.

For the 10 patients undergoing re-operations, the early postoperative GH nadir on OGTT after the second surgery was utilized. IGF-1 was not used in the immediate post-operative remission criteria as the likelihood of normalization to age- and genderadjusted values is low at this time point [16].

Patients with an early GH nadir $>1 \mu \mathrm{g} / \mathrm{L}$ underwent an early post-operative MR scan of the pituitary region within 1-2 weeks of surgery. If residual tumour was identifiable on this scan, the patient was counselled with regard to the option of further transsphenoidal re-exploration. The decision to undertake redo surgery also took account of other factors such as patient age, comorbidity, preexisting hypopituitarism, risk of new pituitary hormone deficiencies, and alternative approaches such as medical therapy for residual disease. In general, redo surgery was undertaken at 2-4 weeks postoperatively. Further assessment of the GH axis was undertaken at 3 and 12 months post-operatively, then at yearly intervals using OGTT (on occasions GH day curve) and/or IGF-1 levels as appropriate.

Disease remission in acromegaly was defined according to the 2010 consensus statement, which defines remission as requiring a $\mathrm{GH}$ nadir $<0.4 \mu \mathrm{g} / \mathrm{L}$ on OGTT, a random $\mathrm{GH}<1 \mu \mathrm{g} / \mathrm{L}$ and a normal age- and sex-adjusted IGF-1 at final follow-up [2]. Patients with discrepancy in the final follow-up, IGF-1 and GH nadir levels (e.g., IGF-1 $>100 \%$ ULN, but GH nadir $<0.4 \mu \mathrm{g} / \mathrm{L}$ or vice versa) were considered not in remission. Disease remission was determined at the last available endocrine assessment (i.e., "final followup") and before commencement of post-operative medical therapy and/or radiotherapy.

All patients also underwent routine post-operative testing for the rest of the pituitary hormones in the form of basal pituitary function tests and a glucagon and/or insulin stress tests at 2-3 weeks post-operatively and again at future time points as required. New-onset post-operative hypopituitarism and the need for any new pituitary hormone replacements were noted.

\section{Statistical Analysis}

Data were processed using SPSS (Chicago, IL) statistical software. Non-parametric data were tested using the Pearson $\chi^{2}$ and Mann-Whitney $U$ tests. Normally distributed parametric data were compared using Student's $t$-test or ANOVA and post hoc Bonferroni tests. Multivariate analysis was performed using logistic regression to assess the influence of various factors on disease remission.

\section{Results}

\section{Patient Demographics}

Eighty-one patients were treated with endoscopic transsphenoidal surgery over the study period in Manchester $(N=70)$ and Melbourne $(N=11)$. As there were no significant differences in baseline patient characteristics or longer term disease remission, the data from both centres were pooled.

There were 41 females and 40 males, with an overall mean \pm SD age of $49 \pm 14$ years (range 25-77 years). Overall, 5/81 (6\%) patients were diagnosed with diabetes preoperatively. All patients had a minimum of 12 months biochemical follow-up with a mean \pm SD follow-up duration of $44 \pm 25$ months (range 12-108 months). The majority of tumours were macroadenomas $(N=63 ; 78 \%)$, with radiological evidence of invasiveness, as judged by Hardy (i.e., D-E and/or 3-4) or Knosp grades (i.e., 3 or $4)$, noted in 28 (35\%) patients.

Pre-operative medical treatment was given to 15 (19\%) patients, including SSAs in 14 patients and dopamine agonists in 1 patient. At least a 3-month drug washout period was allowed before surgery to enable meaningful interpretation of GH levels post-operatively. 
Table 1. Variables associated with acromegaly disease remission, as defined by the 2010 consensus statement

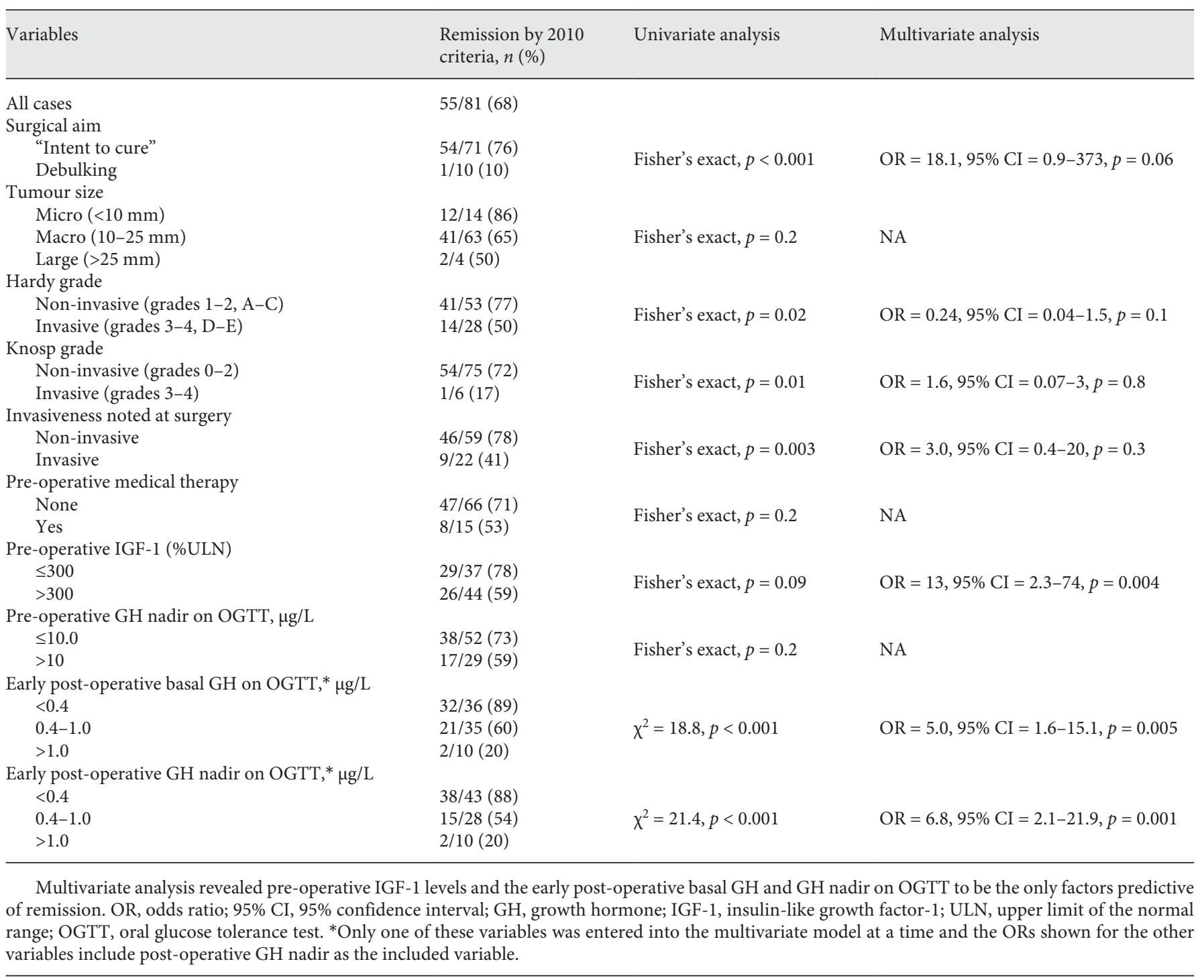

Overall, $71(88 \%)$ patients were treated with intent to cure and $10(12 \%)$ patients had a planned debulking alone due to significant bony and/or cavernous sinus invasion. Ten patients underwent early re-exploration of the pituitary for residual tumour.

\section{Endocrinological Outcome}

Post-operative remission rates based on the 2010 consensus criteria and the various related radiological and biochemical factors are tabulated in Table 1. At final follow-up and before commencement of medical and/or radiotherapy, remission was achieved in 55 (68\%) patients, and this was higher (i.e., $N=54 ; 76 \%$ ) when taking into consideration only those patients operated on an "intent to cure" basis. At final follow-up, 26 (32\%) patients were not in biochemical remission, requiring additional medical and/or radiotherapy in some (see below).

On univariate analysis, there were significant differences in the remission rates between invasive and noninvasive adenomas as determined by the Hardy $(p=0.02)$ and Knosp grades $(p=0.01)$ and as judged intra-operatively by the surgeon ( $p=0.003$; Table 1$)$. A non-significant trend towards lower remission rates was found with a larger tumour size ( $p=0.2$ ), pre-operative IGF- $1>300 \%$ ULN ( $p=0.09)$, pre-operative medical treatment $(p=0.2)$, and pre-operative $\mathrm{GH}$ nadir $>10 \mu \mathrm{g} / \mathrm{L}(p=0.18$; Table 1$)$. 
Table 2. Use of early OGTT as a test of disease remission

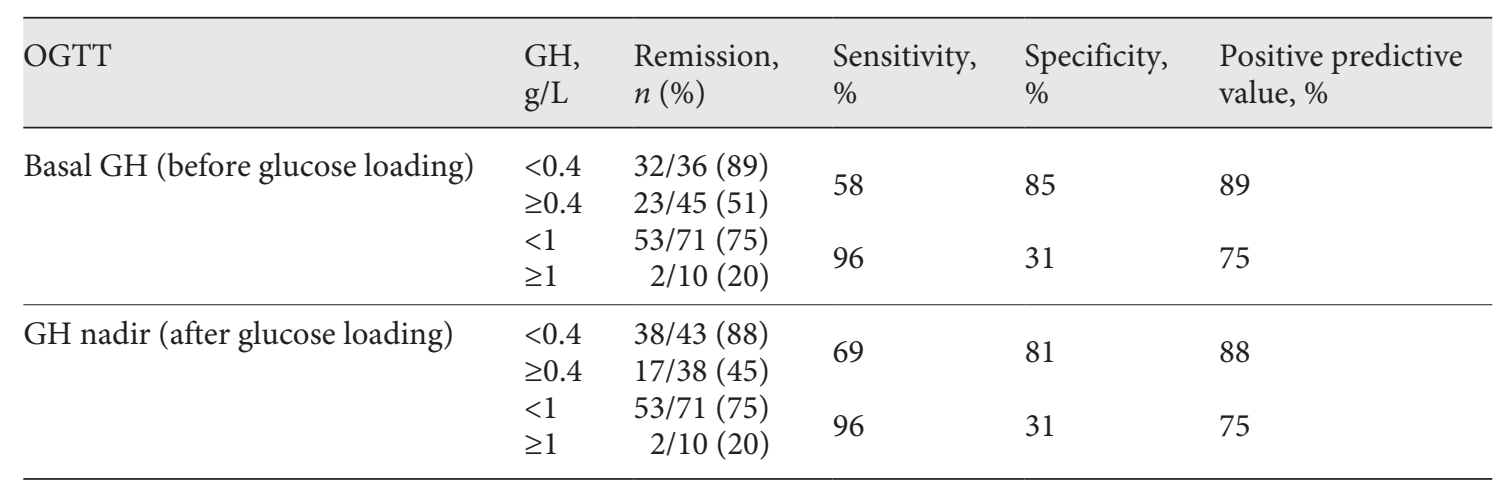

The performance of basal GH levels before glucose loading and the GH nadir after glucose loading is shown. A GH level of $<0.4 \mu \mathrm{g} / \mathrm{L}$ at both time points was superior in terms of specificity and positive predictive value, compared to $<1 \mu \mathrm{g} / \mathrm{L}$. OGTT, oral glucose tolerance test; GH, growth hormone.

On early post-operative testing, a GH nadir on OGTT of $<0.4 \mu \mathrm{g} / \mathrm{L}$ had a higher long-term remission rate (88\%), when compared to GH nadir of $0.4-1 \mu \mathrm{g} / \mathrm{L}(54 \%)$ or $>1$ $\mu \mathrm{g} / \mathrm{L}(20 \% ; p<0.001$; Table 1). The basal GH level (i.e., pre-glucose loading) was separately evaluated, and patients with a basal GH of $<0.4 \mu \mathrm{g} / \mathrm{L}$ had a higher long-term remission rate (89\%), when compared to basal GH of $0.4-$ $1 \mu \mathrm{g} / \mathrm{L}(60 \%)$ or $>1 \mu \mathrm{g} / \mathrm{L}(20 \%)(p<0.001$; Table 1$)$. There was a strong correlation between the basal GH pre-glucose loading and the GH nadir after glucose loading during the OGTT (Pearson's coefficient $=0.54, p<0.001$ ).

On multivariate analysis, pre-operative IGF-1 $>300 \%$ $\mathrm{ULN}(\mathrm{OR}=13,95 \% \mathrm{CI}=2.3-74, p=0.004)$, early postoperative $\mathrm{GH}$ nadir $>0.4 \mu \mathrm{g} / \mathrm{L}(\mathrm{OR}=6.80,95 \% \mathrm{CI}=2.1-$ $21.9, p=0.001)$, and basal GH pre-glucose loading $>0.4$ $(\mathrm{OR}=5.0,95 \% \mathrm{CI}=1.6-15.1, p=0.005)$ were associated with residual disease, not meeting remission criteria in acromegaly (Table 1 ). As a test of disease remission, a basal GH level or GH nadir of $<0.4 \mu \mathrm{g} / \mathrm{L}$ on early postoperative OGTT tests had better specificity and positive predictive value compared to $<1 \mu \mathrm{g} / \mathrm{L}$ (Table 2 ). The early OGTT was well tolerated by all patients, with no adverse events noted.

The changes in GH nadir on OGTT for individual patients with respect to the early post-operative GH nadir are shown in Figure 1. Of patients with an early post-operative $\mathrm{GH}$ nadir $<0.4 \mu \mathrm{g} / \mathrm{L}(N=43)$, at final follow-up, 38 were in remission and 5 had borderline results with discrepant GH nadir and IGF-1 values. In patients with an early post-operative $\mathrm{GH}$ nadir between 0.4 and $1 \mu \mathrm{g} / \mathrm{L}$ $(N=28)$, at final follow-up, 15 were in remission, 6 were not in remission, and 7 had borderline results with dis- crepant GH nadir and IGF-1 values. In patients with an early post-operative $\mathrm{GH}$ nadir $>1 \mu \mathrm{g} / \mathrm{L}(N=10)$, at final follow-up, 2 were in remission, 7 were not in remission, and 1 patient had borderline results with discrepant $\mathrm{GH}$ nadir and IGF-1 values. Overall, 13 (16\%) patients had discrepancy between the IGF-1 and GH nadir results, with a GH nadir $<0.4 \mu \mathrm{g} / \mathrm{L}$, but raised IGF-1 $(N=10)$, or with a normal IGF-1, but persistently raised GH nadir $>0.4 \mu \mathrm{g} / \mathrm{L}(\mathrm{N}=3)$.

Figure 2 demonstrates the temporal changes in IGF-1 (Fig. 2a) and GH nadir on OGTT (Fig. 2b) in all patients $(N=81)$, in only patients in long-term remission $(N=55)$ and those not in remission $(N=26)$. The post-operative decrease in GH nadir levels on OGTT was rapid in reaching the lowest point in days, in contrast to the drop in post-operative IGF-1 levels, which were more gradual, reaching the lowest point at 1-2 years after surgery (Fig. 2).

\section{Post-Operative Complications and Interventions}

Overall, $16(20 \%)$ patients developed new pituitary hormone deficiency post-operatively, including ACTH deficiency requiring hydrocortisone $(N=8)$, TSH deficiency requiring thyroxine $(N=4)$, gonadotropin deficiency $(N=7)$, and permanent diabetes insipidus, requiring DDAVP replacement $(N=6)$. Other post-operative complications included CSF leak and meningitis $(N=1)$, syndrome of inappropriate $\mathrm{ADH}$ secretion $(N=1)$, and a patient with persistent right facial pain that resolved following a microvascular decompression procedure 4 years later. 


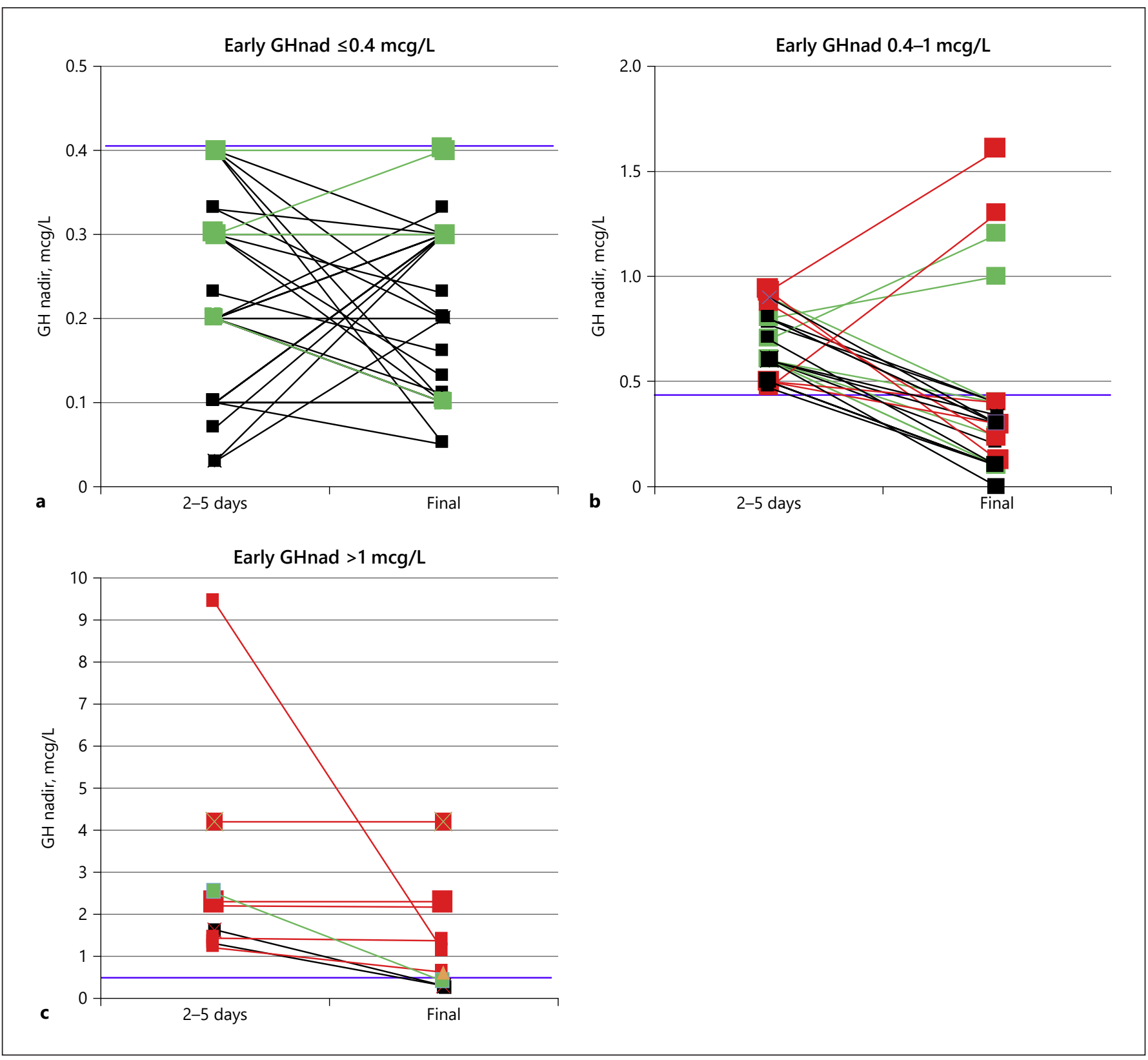

Fig. 1. Line charts depicting the trends in GHnadir on OGTT $(\mu \mathrm{g} / \mathrm{L})$ for individual patients, with respect to early (day $2-5)$ postoperative GH nadir values categorized in terms of $<0.4(n=43)$ (a), $0.4-1.0(n=28)(\mathbf{b})$ and $>1.0 \mu \mathrm{g} / \mathrm{L}(n=10)(\mathbf{c})$. Black lines depict patients in remission; green lines depict those with borderline results (i.e., with discrepant post-operative IGF-1 and GH nadir val-

At final follow-up, 26 (32\%) patients were not in biochemical remission. For patients with residual GH excess and relevant clinical symptoms $(N=14)$, further treatment had been commenced with SSA $(N=8)$, SSA and dopamine agonists $(N=2)$, dopamine agonists $(N=1)$,

ues); and red lines depict those patients not in remission according to the 2010 consensus criteria [2]. Blue reference line refers to the $0.4 \mu \mathrm{g} / \mathrm{L} \mathrm{GH}$ nadir cut-off used to define remission in the 2010 consensus statement. GH, growth hormone; OGTT, oral glucose tolerance test; IGF-1, insulin-like growth factor-1.

and SSA and radiotherapy $(N=3)$. In 12 patients, the biochemical evidence of $\mathrm{GH}$ excess was being managed conservatively as there were no relevant clinical symptoms. 


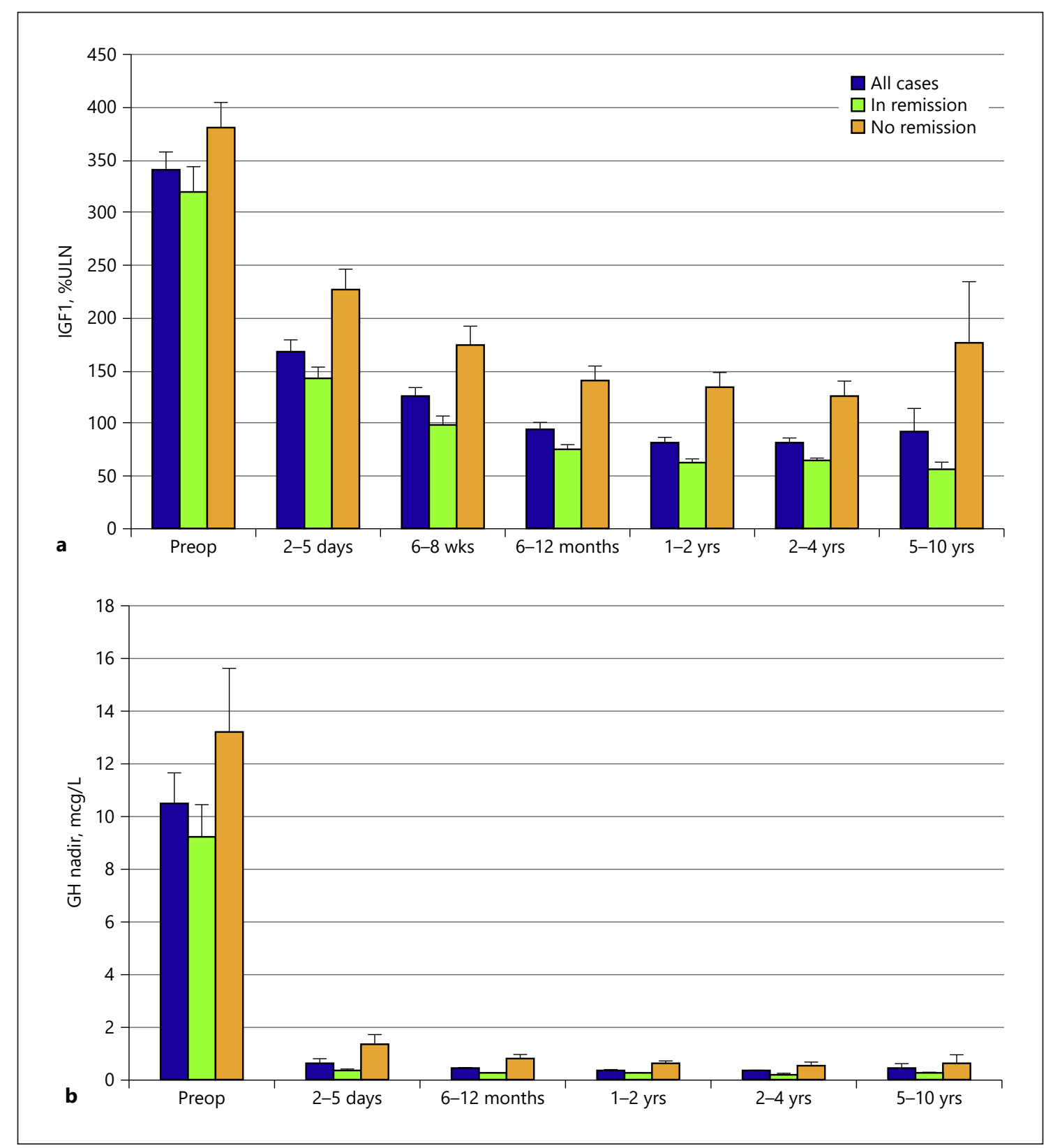

Fig. 2. Bar chart depicting the trends in IGF-1 (a) and GH nadir (b) values on OGTT from pre-operative to various time points post-operatively. The mean values for all cases $(N=81)$, those in remission $(N=55)$, and not in remission $(N=26)$ are demonstrated. The IGF-1 is expressed as \%ULN (mean \pm standard error of

\section{Redo Surgery}

Ten patients underwent early re-exploration for residual disease, given that early post-operative OGTT and post-operative MR imaging were suggestive of residual tumour. These patients were also counselled with respect to alternatives such as conservative (i.e., wait mean) for an age- and sex-matched control population. The GH nadir is expressed as the mean \pm standard error of mean in $\mu \mathrm{g} / \mathrm{L}$. $\mathrm{GH}$, growth hormone; IGF-1, insulin-like growth factor-1; OGTT, oral glucose tolerance test; ULN, upper limit of the normal range.

and see) or appropriate medical therapy. The tumour characteristics and biochemical test results of all patients (Table 3 ) and the pre- and early post-operative MR scans of 2 case examples (cases 2 and 8 , Table 3 ) are shown in Figure 3. 

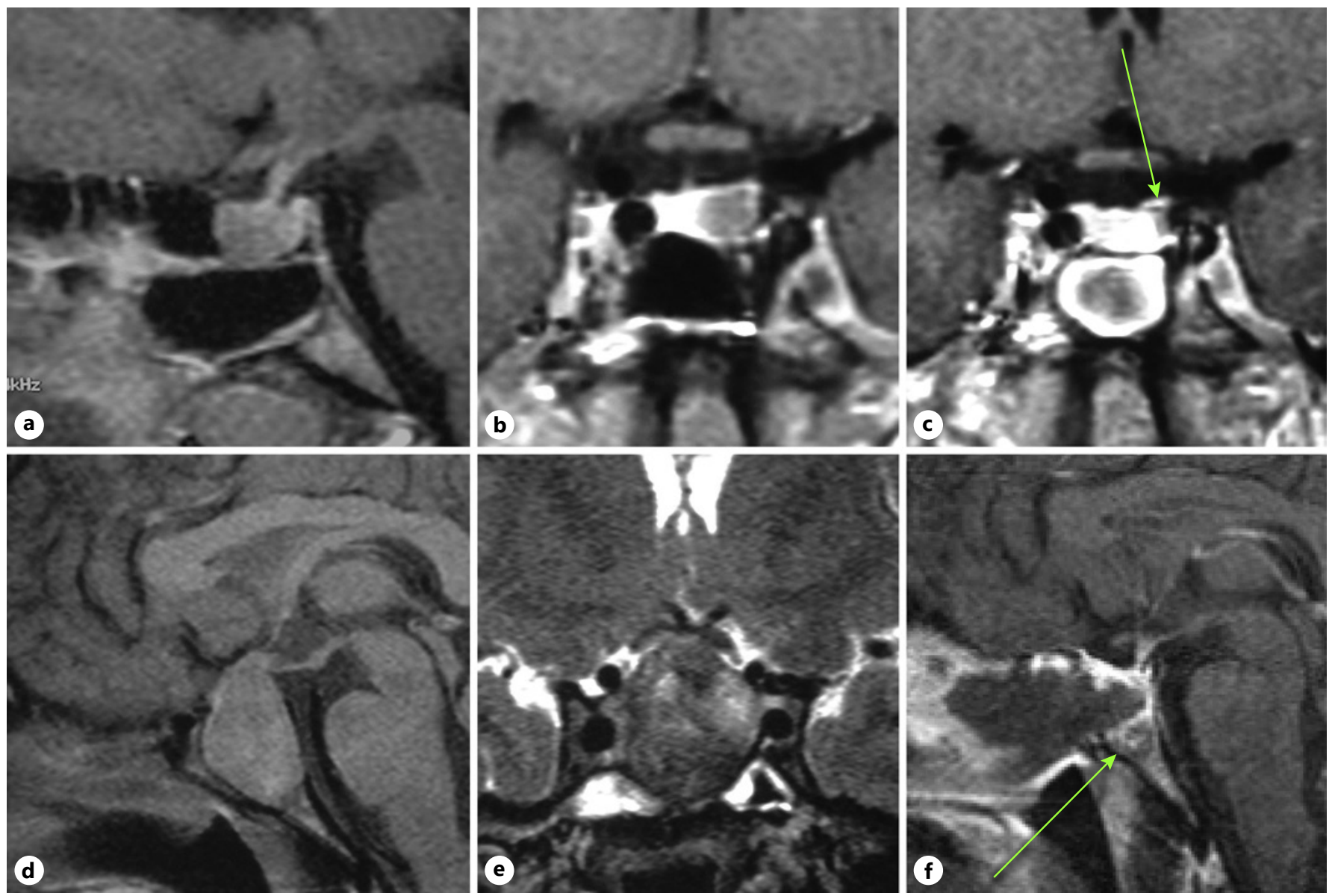

Fig. 3. MR scans depicting pre-operative (a, b, d, e) and 2-week post-operative (c, f) MR scans after transsphenoidal surgery in a 31-year-old (a, b, c; case 2 in Table 3 ) and a 37-year-old male patient (d, e, f; case 8 in Table 3). Sagittal T1-weighted (a, d) and coronal T2-weighted (e) MR images and gadolinium-enhanced coronal T1-weighted (b, c) and sagittal T1-weighted (f) images are shown. Residual tumour adjacent to the left cavernous carotid artery (c) and tumour invading the bony clivus (f) are indicated by green arrows.
Following the re-operation, there was reduction in the repeat early post-operative $\mathrm{GH}$ nadir levels in 9 patients and 5 (50\%) went into remission at final follow-up. One patient had borderline results with a raised GH nadir of $1.2 \mu \mathrm{g} / \mathrm{L}$ and a normal IGF-1 (i.e., $56 \%$ of ULN) at 30-month follow-up (case 5, Table 3). There was histological confirmation of residual somatotroph tumour in 5 patients and possible GH hyperplasia in 1 patient (Table 3$)$.

In patients undergoing redo surgery, at final followup, post-operative hypopituitarism (i.e., as defined by new pituitary hormone deficiency) was noted in 6(60\%) patients (Table 3). This risk was greater compared to those patients having a single transsphenoidal procedure (i.e., 10 of 71 patients; $14 \%$; $p<0.001 ; \chi^{2}$ test). Re- operated patients who were not in remission at final follow-up were commenced on SSAs $(N=4)$ or dopamine agonists $(N=1)$ or were being observed $(N=6$; Table 3).

\section{Discussion}

Biochemical remission in patients with acromegaly reduces GH-related morbidity while maintaining life expectancy. The remission rate after surgery alone is reported to be between 27 and $80 \%[17,18]$. Variables influencing surgical remission rate include the criteria used to define remission and factors related to imaging (e.g. tumour size and invasiveness) and endocrine (e.g., pre-op- 


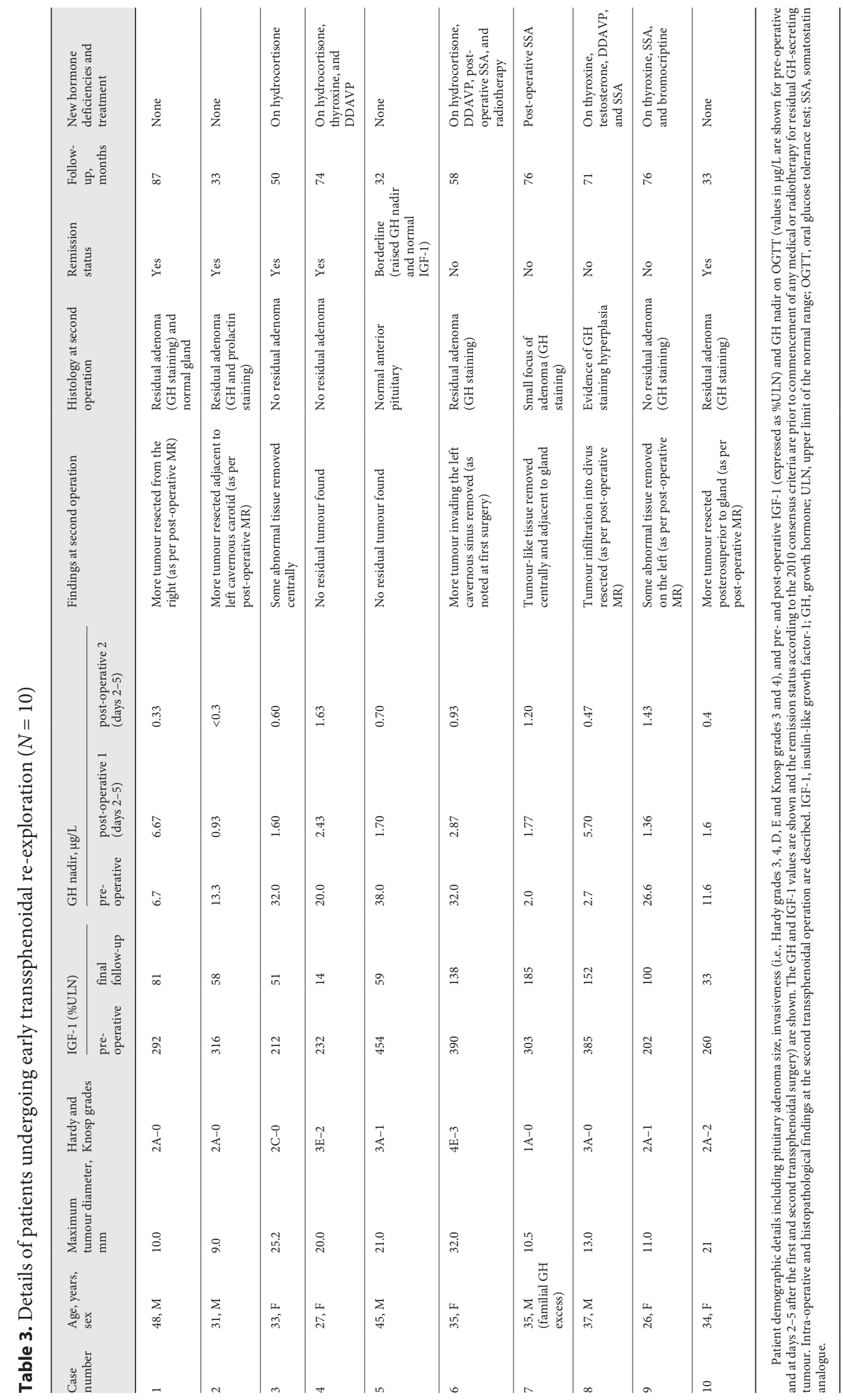


erative GH levels), and operative parameters, notably surgeon experience $[17,18]$. Where biochemical remission is not achieved, early identification of patients with residual disease is important for prognostication purposes and to offer further treatment including timely surgical re-exploration in selected patients.

At 2 centres specializing in pituitary surgery, an overall remission rate of $68 \%$ was achieved at a mean follow-up period of 44 months. About one-third of the tumours were invasive. Multivariate analysis revealed that pre-operative high IGF-1 levels (i.e., $>300 \%$ ULN) and the early post-operative basal and nadir GH on OGTT were significant predictors of longer term remission. Thus, we noted that a basal and nadir $\mathrm{GH}$ of $\leq 0.4 \mu \mathrm{g} / \mathrm{L}$ at $2-5$ days post-operatively were associated with long-term remission in 89 and $88 \%$ of patients, respectively, with the remaining patients failing to be in remission due to discrepant GH and IGF-1 results.

Furthermore, a relatively high GH nadir on the early post-operative OGTT was an important indicator of residual disease. Thus, in our study, $80 \%$ of patients with a GH nadir $>1 \mu \mathrm{g} / \mathrm{L}$ and $46 \%$ of patients with $\mathrm{GH}$ nadir between 0.4 and $1.0 \mu \mathrm{g} / \mathrm{L}$ failed to achieve long-term remission according to the 2010 consensus statement. However, a significant proportion (i.e., $45 \%$ ) of patients with an early post-operative $\mathrm{GH}$ nadir level of $>0.4 \mu \mathrm{g} / \mathrm{L}$ can still go into remission longer term. Given the very short half-life of circulating GH (i.e., 20-30 min), the reasons for the delayed remission are not clear. It is possible that the gradual reduction in $\mathrm{GH}$ levels may reflect reduction in the body's stress response to surgery and/or spontaneous necrosis of a few remaining tumour cells after surgery [8]. An early post-operative GH nadir in the range of $0.4-$ $1 \mu \mathrm{g} / \mathrm{L}$ represents a contentious area in this respect and unless there was a clear tumour residuum on the early post-operative MR scan, we opted for close monitoring, with repeat tests of the $\mathrm{GH}$ axis.

Moreover on OGTT, there was a strong correlation between the basal GH level (i.e., pre-glucose loading) and the GH nadir after administration of glucose. The sensitivity, specificity, and positive predictive value of these tests were similar in terms of predicting disease remission. Thus, a basal fasting GH level can also be a reliable alternative to GH nadir on an early post-operative OGTT as an indicator of long-term remission and residual disease.

The predictive value of an early post-operative $\mathrm{GH}$ on achieving long-term remission in acromegaly has been investigated in other recent studies, although the results are somewhat variable [6-9]. Rotermund et al. [9] re- ported a $100 \%$ rate of remission at 16 -month mean follow-up in 14 patients with a GH nadir of $<1 \mu \mathrm{g} / \mathrm{L}$ on post-operative days $2-4$. Sarkar et al. [8] followed up 86 patients for a mean follow-up period of 27.5 months and found that the post-operative day $7 \mathrm{GH}$ nadir on OGTT was predictive of remission in multivariate analysis. The authors also found that the sensitivity/specificity of a day 1 postoperative 8 a.m. GH gave similar sensitivity to the day 7 value. Kim et al. [7] found that remission at 3.8 -year mean follow-up was maintained in $125 / 127 \mathrm{pa}$ tients $(98.4 \%)$ with a $\mathrm{GH}$ nadir of $<1 \mu \mathrm{g} / \mathrm{L}$ on post-operative day 7. Kristof et al. [6] noted that the post-operative day $14 \mathrm{GH}$ nadir on OGTT correctly predicted final endocrine outcome in $82.9 \%$ of patients at 5 -year mean follow-up. Interestingly, the value of intraoperative GH levels has also been assessed during transsphenoidal surgery in patients with acromegaly. In one such study, 28 patients underwent measurement of GH levels at serial time points after adenoma removal [19]. Use of the GH levels obtained was limited in at least 2 ways. First, the $\mathrm{GH}$ level fell successively at 10 -min intervals in patients with and without successful surgery for up to the first 180 min post-adenoma removal, making it difficult to define absolute thresholds for cure. Second, GH levels obtained 60-120 min and 90-180 min post-adenoma removal had lower sensitivity and specificity rates than those obtained 1 week postoperatively [19]. Thus, the practical challenges and cost of obtaining intra-operative GH levels do not appear to justify the benefits. Despite these early reports, there remains no consensus as to the ideal timing for the early post-operative GH test, the type of $\mathrm{GH}$ test (i.e., random $\mathrm{GH}$ levels, $\mathrm{GH}$ nadir on OGTT, or mean GH day curve), and the best cut-off value for the GH [10].

The reasons for a subtotal surgical resection of pituitary adenoma include the large size of adenoma and extra-sellar invasion notably into the cavernous sinus and bony clivus $[17,18]$. Even in expert surgical hands and with the improved access and vision offered by modern angled endoscopes, residual tumour is likely in a small subgroup of patients. Prompt identification of this subgroup can be useful as early transsphenoidal re-exploration is technically less demanding, given the reduced risk of post-operative fibrosis [5].

In the present study, the identification of patients with residual tumour was based on a combination of biochemical and radiological findings. Thus, in addition to the early tests of the GH axis, we undertook early post-operative MR imaging for patients with relatively high early post-operative GH nadir levels on OGTT (i.e., $>1 \mu \mathrm{g} / \mathrm{L}$ ). 
Residual adenoma was suspected on the early post-operative MR scans in 10 such patients who underwent surgical re-exploration within 4 weeks from the first surgery. For this subgroup of patients, there was histological confirmation of residual GH staining adenoma in only 5 and possible GH hyperplasia in 1 patient, but interestingly, there was reduction in GH nadir levels in 9 patients. Interpretation of the early post-operative imaging can be difficult in some patients due to the presence of post-operative blood in the tumour bed and artefacts from the haemostatic and other repair material used at the time of surgery. Such early MR imaging should also be interpreted in the context of preoperative imaging as well as operative findings. Others have explored the use of intraoperative MR in such cases and reported that the intraoperative detection of residual tumour on MR imaging led to a $5 \%$ increase in remission rate in patients undergoing transsphenoidal surgery for acromegaly [20]. However, interpretation of the intra-operative images, particularly from low-field MR images, and the additional expense and delays incurred with use of an intra-operative MR scanner remain major limitations to more routine use of this technology.

The decision to re-operate in patients suspected to have residual adenoma may not be straightforward. In addition to the biochemical and radiological parameters, several other factors need to be considered before re-exploring patients suspected to have residual adenoma. In this respect, the surgical accessibility of residual tumour is an important factor to consider. For example, the risks of re-exploration may not be justified if residual tumour is difficult to access, particularly in relation to critical neurovascular structures such as the carotid artery and invasion into the cavernous sinus. Furthermore, with a second operation, the patients need counselling about the lower chance of success (i.e., $50 \%$ remission with re-exploration), higher risk of hypopituitarism (i.e., 60 vs. 14\%), and other potential complications such as CSF leak, although these were not evident in this small series. Other factors to take into consideration include the patient's age, comorbidity, the risk to fertility, the extent of hypopituitarism after the first surgery, and the alternative of medical treatment for GH excess. However, in economic terms, there may be a significant financial benefit in achieving remission in acromegaly by surgical re-exploration compared to long-term medical management of residual GH excess [21]. Thus, there remains a need for careful and detailed discussion with patients, outlining the risks involved with a second procedure and other viable alternatives such as medical therapy and radiotherapy to address residual adenoma.

In contrast to the usefulness of early GH levels in predicting long-term remission, IGF-1 levels took 1-2 years to stabilize following surgery. The 2010 consensus statement recommends measurement of IGF-1 levels after at least 12 weeks post-operatively [10]. However, our results suggest that even at this time point, IGF-1 should be interpreted with caution. Moreover, Shin et al. [22] measured IGF-1 levels serially on acromegaly patients undergoing surgery and found that normalization of IGF-1 levels took up to 57 months, with $41 \%$ of patients taking longer than 3 months. The reason for the lengthy delay in normalization of IGF-1 levels is unclear and is not solely dependent on its circulating half-life of $12-15 \mathrm{~h}$. Other potential reasons include the $\mathrm{GH}$-independent synthesis of IGF-1 owing to autonomy [23]. It may also reflect a slow transition from a relatively $\mathrm{GH}$-resistant body state towards normalization of GH sensitivity accompanied by changes in the pulsatility of GH release and GH-binding protein levels until a new equilibrium is established between GH secretion and responsiveness [23].

In present study, a discrepancy between IGF-1 levels and GH nadir was seen in $16 \%$ of the post-operative patients with acromegaly at final follow-up. In a recent meta-analysis of acromegaly patients, such discrepancy between the post-operative IGF-1 and GH results was observed in $25.7 \%$ of patients [24]. The predominant format was raised IGF-1 with normal GH levels and a higher discordance has been linked to treatment with SSAs, the increased use of ultrasensitive $\mathrm{GH}$ assays, and the changes in the IGF-1 assays from Immulite to IDS iSYS type $[24,25]$. The implications of the discordance between $\mathrm{GH}$ and IGF-1 remain unclear, but as per the consensus guidelines, these patients are considered not in remission. However, in this patient subgroup, additional medical treatment is usually instituted in patients with residual or recurrent symptoms of GH excess [5]. It is therefore essential that both IGF-1 and GH levels are measured in evaluating remission from acromegaly in these patients.

Limitations of the present study include the disproportionate number of patients from one of the centres. However, there were no significant differences in demographics between the pooled cohorts. Although our follow-up period was longer than most studies, long-term endocrine follow-up is essential, given the small risk of tumour recurrence longer term. 


\section{Conclusions}

In the present study of endoscopic transsphenoidal surgery for acromegaly, the use of early basal or nadir $\mathrm{GH}$ on OGTT is predictive of long-term remission in surgically operated cases of acromegaly. Furthermore, this test can help identify those with likely residual adenoma who can be considered for early surgical re-exploration with the aim of disease remission. However, patient selection for surgical re-exploration may not be straightforward, and patients will need counselling with regard to the increased risk of hypopituitarism and the availability of alternative options such as medical management.

\section{Acknowledgements}

The authors have no acknowledgements.

\section{Statement of Ethics}

This study received ethical approval by the Health Research Authority (Integrated Research Application System ID 261250). In addition, Institutional Review Board approval was obtained by each institute. Informed written consent was not deemed necessary by the Health Research Authority based on the study design.

\section{Conflict of Interest Statement}

The authors have no conflicts of interest to declare.

\section{Funding Sources}

The authors did not receive any funding.

\section{Author Contributions}

Study design and protocol: Y.Y.W., K.K.G., F.R., C.H., P.T., and J.D. Data collection: Y.Y.W., M.W., C.C., K.K.G., T.K., and A.A.-Z. Data analysis: Y.Y.W., M.W., C.C., K.K.G., and T.K. Manuscript write-up: Y.Y.W., M.W., K.K.G., and J.D. Manuscript review and approval: all authors.

\section{References}

1 Giustina A, Barkan A, Casanueva FF, Cavagnini F, Frohman L, Ho K, et al. Criteria for cure of acromegaly: a consensus statement. J Clin Endocrinol Metab. 2000 Feb;85(2):5269.

2 Giustina A, Chanson P, Bronstein MD, Klibanski A, Lamberts S, Casanueva FF, et al. A consensus on criteria for cure of acromegaly. J Clin Endocrinol Metab. 2010 Jul;95(7): 3141-8.

3 Yamada S, Aiba T, Takada K, Ozawa Y, Shimizu T, Sawano S, et al. Retrospective analysis of long-term surgical results in acromegaly: preoperative and postoperative factors predicting outcome. Clin Endocrinol. 1996 Sep; 45(3):291-8.

4 Gittoes NJ, Sheppard MC, Johnson AP, Stewart PM. Outcome of surgery for acromegaly: the experience of a dedicated pituitary surgeon. QJM. 1999 Dec;92(12):741-5.

5 Wang YY, Higham C, Kearney T, Davis JR, Trainer P, Gnanalingham KK. Acromegaly surgery in Manchester revisited: the impact of reducing surgeon numbers and the 2010 consensus guidelines for disease remission. Clin Endocrinol. 2012 Mar;76(3):399-406.
6 Kristof RA, Grote A, Redel L, Neuloh G, Klingmüller D, Schramm J. The common consensus criteria have high predictive values for long-term postoperative acromegaly remission. Acta Neurochir. 2011 Jan;153(1):19-25.

7 Kim EH, Oh MC, Lee EJ, Kim SH. Predicting long-term remission by measuring immediate postoperative growth hormone levels and oral glucose tolerance test in acromegaly. Neurosurgery. 2012 May;70(5):1106-13; discussion 13.

8 Sarkar S, Jacob KS, Pratheesh R, Chacko AG. Transsphenoidal surgery for acromegaly: predicting remission with early postoperative growth hormone assays. Acta Neurochir. 2014 Jul;156(7):1379-87; discussion 87.

9 Rotermund R, Burkhardt T, Rohani Z, Jung R, Aberle J, Flitsch J. Value of early postoperative random growth hormone levels and nadir growth hormone levels after oral glucose tolerance testing in acromegaly. Growth Horm IGF Res. 2018 Aug;41:64-70.

10 Melmed S, Bronstein MD, Chanson P, Klibanski A, Casanueva FF, Wass JAH, et al. A consensus statement on acromegaly therapeutic outcomes. Nat Rev Endocrinol. 2018 Sep;14(9):552-61.

11 Trainer PJ, Barth J, Sturgeon C, Wieringaon G. Consensus statement on the standardisation of GH assays. Eur J Endocrinol. 2006 Jul; 155(1):1-2.
12 Hardy J. Transphenoidal microsurgery of the normal and pathological pituitary. Clin Neurosurg. 1969;16:185-217.

13 Knosp E, Steiner E, Kitz K, Matula C. Pituitary adenomas with invasion of the cavernous sinus space: a magnetic resonance imaging classification compared with surgical findings. Neurosurgery. 1993 Oct;33(4):610-8; discussion 17-8.

14 Micko AS, Wöhrer A, Wolfsberger S, Knosp E. Invasion of the cavernous sinus space in pituitary adenomas: endoscopic verification and its correlation with an MRI-based classification. J Neurosurg. 2015 Apr;122(4):80311.

15 Leach P, Abou-Zeid AH, Kearney T, Davis J, Trainer PJ, Gnanalingham KK. Endoscopic transsphenoidal pituitary surgery: evidence of an operative learning curve. Neurosurgery. 2010 Nov;67(5):1205-12.

16 Kreutzer J, Vance ML, Lopes MB, Laws ERJr. Surgical management of GH-secreting pituitary adenomas: an outcome study using modern remission criteria. J Clin Endocrinol Metab. 2001 Sep;86(9):4072-7.

17 Lissett CA, Peacey SR, Laing I, Tetlow L, Davis JR, Shalet SM. The outcome of surgery for acromegaly: the need for a specialist pituitary surgeon for all types of growth hormone $(\mathrm{GH})$ secreting adenoma. Clin Endocrinol. 1998 Nov;49(5):653-7. 
18 Jho HD. Endoscopic transsphenoidal surgery. J Neurooncol. 2001 Sep;54(2):187-95.

19 Valdemarsson S, Ljunggren S, Cervin A, Svensson C, Isaksson A, Nordström CH, et al. Evaluation of surgery for acromegaly: role of intraoperative growth hormone measurement? Scand J Clin Lab Invest. 2001;61(6): $459-70$.

20 Bellut D, Hlavica M, Schmid C, Bernays RL. Intraoperative magnetic resonance imagingassisted transsphenoidal pituitary surgery in patients with acromegaly. Neurosurg Focus. 2010 Oct;29(4):E9.

21 Wilson LS, Shin JL, Ezzat S. Longitudinal assessment of economic burden and clinical outcomes in acromegaly. Endocr Pract. 2001 May-Jun;7(3):170-80.

22 Shin MS, $\mathrm{Yu} \mathrm{JH}$, Choi JH, Jung $\mathrm{CH}$, Hwang JY, Cho YH, et al. Long-term changes in serum IGF-1 levels after successful surgical treatment of growth hormone-secreting pituitary adenoma. Neurosurgery. 2013 Sep; 73(3):473-9; quiz 79.

23 Feelders RA, Bidlingmaier M, Strasburger CJ, Janssen JA, Uitterlinden P, Hofland LJ, et al. Postoperative evaluation of patients with acromegaly: clinical significance and timing of oral glucose tolerance testing and measurement of (free) insulin-like growth factor I, acid-labile subunit, and growth hormone-bind- ing protein levels. J Clin Endocrinol Metab. 2005 Dec;90(12):6480-9.

24 Kanakis GA, Chrisoulidou A, Bargiota A, Efstathiadou ZA, Papanastasiou L, Theodoropoulou A, et al. The ongoing challenge of discrepant growth hormone and insulin-like growth factor I results in the evaluation of treated acromegalic patients: a systematic review and meta-analysis. Clin Endocrinol. 2016 Nov;85(5):681-8.

25 Varewijck AJ, Lamberts SW, van der Lely AJ, Neggers SJ, Hofland LJ, Janssen JA. The introduction of the IDS-iSYS total IGF- 1 assay may have far-reaching consequences for diagnosis and treatment of GH deficiency. J Clin Endocrinol Metab. 2015 Jan;100(1):309-16. 